\title{
Hyperbilirubinemia and Intermittent Lower Urinary Tract Dysfunction: A Story
}

\author{
Viroj Wiwanitkit \\ Received: 5 January 2011 / Accepted: 4 February 2011 /Published online: 5 March 2011 \\ (C) Dr. K C Chaudhuri Foundation 2011
}

Sir,

I read the recent publication on hyperbilirubinemia and urinary tract dysfunction with great interest [1]. In this work, Stojanović et al. concluded that "hyperbilirubinemia might be a predisposing factor for intermittent lower urinary tract dysfunction in children [1]." I have some discussions on this work. First, this is not a case-control but a descriptive study. Hence, it might not be possible to bring any summary on the "risk". Second, the quality control of the laboratory analysis for bilirubin should be discussed. Third, there is no possible pathophysiological explanation for the induction of urinary tract dysfunction by hyperbilirubinemia. Generally, in a case with bilirubin in urine detected by standard urinalysis, there is no report on any effect of bilirubin on urinary function. Conclusively, the work might be only an accidental finding without any clinical importance.

\section{Reference}

1. Stojanović V, Vukavić T. Hyperbilirubinemia and Intermittent Lower Urinary Tract Dysfunction. Indian J Pediatr. 2011 doi:10.1007/s12098-010-0346-0.

\section{Author's Reply}

Sir,

In our study, there were not a significant number of patients with Gilbert's syndrome with pathological uroflow along with the pathological values of bilirubin, but we found interesting and valuable fact that they all had normal uroflow when the values of bilirubin in the serum were normalized. Each patient was a control of its own, postfasting uroflowmetry being experimental and post hypercaloric uroflowmetry, the control.

\footnotetext{
V. Wiwanitkit $(\bowtie)$

Wiwanitkit house, Bangkhae,

Bangkok, Thailand 10160

e-mail: wviroj@yahoo.com
}

As said, this was the first study working on this issue. A number of references were quoted where the authors have pointed out a possible relaxed effect of bilirubin on smooth muscles of gastric fundus and blood vessels $(1,2,3,4)$ and urinary tract muscles $(5,6,7)$. It is proposed that bilirubin expressed the mentioned effect on smooth muscles by potentiating influence on $\mathrm{NO}$ relaxing effect.

It is possible that other diseases except hyperbilirubinemia affect muscle relaxation of urinary tract (i.e. hyperglycemia), which is also mentioned in the article. Further examinations are required to confirm our conclusions.

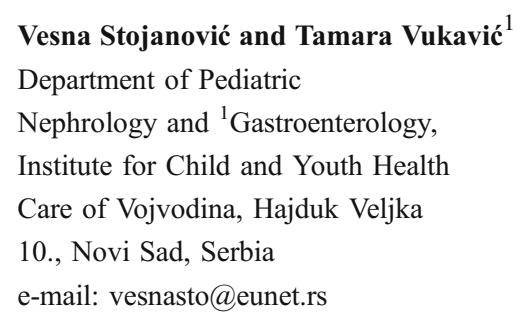

\section{References}

1. Pflueger A, Croatt AJ, Peterson TE, et al. The hyperbilirubinenic Gunn rat is resistant to the pressure effects of angiotensin II. Am J Physiol Renal Physiol. 2005;288:F552-8.

2. Vitek L, Novotny L, Sprel M, Holaj R, Spacil J. The inverse association of elevated serum bilirubin levels with subclinical carotid atherosclerosis. Cerebrovasc Dis. 2006;21:408-14.

3. Erdogan D, Gullu H, Yildirim E, et al. Low serum bilirubin levels are independently and inversely related to impaired flow-mediated vasodilation and increased carotid intima-media thickness in both men and women. Atherosclerosis. 2006;184:431-7.

4. Kawamura K, Ishikawa K, Wada Y, et al. Bilirubin from heme oxygenase-1 attenuates vascular endothelial activation and dysfunction. Arterioscler Thromb Vasc Biol. 2005;25:155-60.

5. Murat N, Kasap B, Kavukcu S, Soylu A, Turkmen M, Gidener S. In vitro analysis of the effect of hyperbilirubinemia on rabbit ureter and bladder. Pediatr Nephrol. 2006;21:328-32.

6. Sarici SU, Kul M, Alpay F. Neonatal jaundice coinciding with or resulting from urinary tract infection? Pediatrics. 2003;112:1212-3.

7. Ghaemi S, Fesharaki RJ, Kelishadi R. Late onset jaundice and urinary tract infection in neonates. Indian J Pediatr. 2007;74:139-41. 